\title{
Anisotropic neutron stars and perfect fluid's energy conditions
}

\author{
A. M. Setiawan ${ }^{1, \mathrm{a}}{ }^{\mathbb{D}}, \mathrm{A}$. Sulaksono ${ }^{2, \mathrm{~b}}$ \\ ${ }^{1}$ Program Studi Pendidikan IPA FMIPA Universitas Negeri Malang, Jl. Semarang 5, Malang 65145, Indonesia \\ ${ }^{2}$ Departemen Fisika FMIPA Universitas Indonesia, Kampus UI, Depok 16424, Indonesia
}

Received: 13 August 2019 / Accepted: 29 August 2019 / Published online: 11 September 2019

(C) The Author(s) 2019

\begin{abstract}
It is reported (Estevez-Delgado and EstevezDelgado in Eur Phys J C 78:673, 2018) recently, that the absence of anisotropic in compact object pressure leads to a solution which is not physically acceptable due to the energy density and speed of sound can not be positive at the origin at the same time. Here, we calculate the pressure and energy density of NSs using realistic EOS predicted by a relativistic mean-field model including hyperons for isotropic and three different anisotropic pressure models such as the one of Doneva and Yazadjiev (Phys Rev D 85:124023, 2012), the one of Herrera and Barreto (Phys Rev D 88:084022, 2013), and the one of Bowers and Liang (Astrophys J 188:657, 1974). We obtain that the all of energy conditions of perfect fluid are satisfied not only by anisotropic NSs profiles but also the one of isotropic NSs.
\end{abstract}

\section{Introduction}

The dynamics of compact objects such as neutron stars (NSs) depend on the matter and gravitational theories. To limit theoretical possibility, one usually use general relativity (GR) as a standard gravitational theory. While the matter is modeled by a perfect ideal fluid with the pressure distributed isotropically. However, many studies have shown that the pressure of compact objects could be distributed anisotropically. The anisotropic pressure of matter is due to the matter or geometric properties. The anisotropy pressure may affect cracking or overturning instability, compactness, maximum mass, radius, gravitational redshift, etc. (please see Refs. [1-9] and the references therein for reviews). The discussions of the relation of the instability of anisotropic stars with perfect fluid energy condition have been reported in Refs. [4,10-12]. It is worthy to note that since compactness limit of self-gravitating, isotropic, and spherically symmetric object of mass $M$ and

\footnotetext{
a e-mail: agung.mulyo.fmipa@um.ac.id

b e-mail: anto.sulaksono@sci.ui.ac.id
}

radius $R$ is $M / R=4 / 9$, the existence of ultra-compact objects with compactness close to one of black hole is ruled out. Therefore, recently, there are some studies about anisotropic stars as ultra-compact objects within GR [13,14]. To this end, it is recently reported [15] that through general analytic compact star model construction, it is evidence that the physical effect of anisotropic pressure is crucial. The absence of pressure anisotropy leads to the solution which is not physically acceptable where the energy density and speed of sound can not be positive at the origin at the same time.

NSs are one of the possible end-points of evolutionary high mass stars, and they are also one of the densest objects in the universe. The NSs has a very strong gravity field, and the corresponding NS matter equation of state (EOS) is quite extreme. The NSs EOS could not be different from that observed in the laboratory. Even though significant progress in constraining EOS of NSs from maximum masses and radii that extracted from NS property observations have been made up to now, the EOS of NSs, especially in the inner core is still unknown properly (please see Refs. [16,17] for a recent review and the references therein.). It is also reported that the gravitation wave (GW) signal emitted by compact binary objects will take a vital role in the future for understanding NSs properties [18]. Furthermore, the first observation of GWs from a binary NSs inspiral known as GW170817 can provide a stringent constraint on EOS of NSs models [19,20]. We also need to note that the possibility of "hyperionization puzzle" in the inner core of NSs can be solved by considering anisotropic pressure. This issue was discussed in Ref. [2].

In this present work, we will show that the perfect fluids energy conditions is satisfied by isotropic and anisotropic NSs profiles using realistic EOS predicted by relativistic mean-field (RMF) model including hyperons for three different anisotropic pressure models. The anisotropic models that we will investigate are the one of Doneva and Yazadjiev [21], the one of Herrera and Barreto [22], and the one of Bowers and Liang [23]. The results will complement to correspond- 
ing results obtained in Ref. [15] through a concrete example of compact objects, i.e., NSs.

This work is organized as follows. Section 2 we briefly discussed the NSs EOS based on RMF model. Section 3 describes briefly basic equations to calculate NSs properties. Section 4 defines perfect fluid energy conditions used in the analysis. Section 5 is devoted to discussing the results, while the conclusion is given in Sect. 6.

\section{Equation of state anisotropic NSs}

According to it's density profile, a NSs can be classified into inner and outer cores which are occupied the larger parts of NSs and saluted by inner and outer crusts. The outer core consists of nucleons and leptons while the inner core consists of hyperons and or other exotics. The baryons contribution on the equation of state (EOS) of NSs cores can be modeled by RMF model [2]. In RMF models, baryons $(N, \Lambda, \Sigma$, and $\Xi$ ) interacts each other through the exchange of meson fields $(\sigma$, $\omega, \rho$, and $\phi)$. Here, due to $\beta$ stability conditions, lepton should exist. The leptons $(l)$ consist of non-interacting electrons $(e)$ and muon $(\mu)$, respectively [2]. The total Lagrangian density used to calculate EOS of the NSs is [2,24,25],

$\mathcal{L}=\mathcal{L}_{B}^{\text {free }}+\mathcal{L}_{M}^{\text {free }}+\mathcal{L}^{L}+\mathcal{L}^{N L}+\mathcal{L}_{l}^{\text {free }}$,

where the Lagrangian density for free baryons is,

$\mathcal{L}_{B}^{\text {free }}=\sum_{B=N, \Lambda, \Sigma, \Xi} \bar{\Psi}_{B}\left[i \gamma^{\mu} \partial_{\mu}-M_{B}\right] \Psi_{B}$

while Lagrangian density for free meson is,

$$
\begin{aligned}
\mathcal{L}_{M}^{\text {free }}= & \frac{1}{2}\left(\partial_{\mu} \sigma \partial^{\mu} \sigma-m_{\sigma}^{2} \sigma^{2}\right)-\frac{1}{4} \omega_{\mu \nu} \omega^{\mu \nu} \\
& +\frac{1}{2}\left(\partial_{\mu} \sigma^{\star} \partial^{\mu} \sigma^{\star}-m_{\sigma^{\star}}^{2} \sigma^{\star^{2}}\right)+\frac{1}{2} m_{\omega}^{2} \omega_{\mu} \omega^{\mu} \\
& -\frac{1}{4} \phi_{\mu \nu} \phi^{\mu \nu}+\frac{1}{2} m_{\phi}^{2} \phi_{\mu} \phi^{\mu}-\frac{1}{4} \rho_{\mu \nu} \rho^{\mu \nu} \\
& +\frac{1}{2} m_{\rho}^{2} \rho_{\mu} \rho^{\mu} .
\end{aligned}
$$

The Lagrangian density for linear couplings of baryon and mesons can be written as,

$$
\begin{aligned}
\mathcal{L}^{L}= & \sum_{B=N, \Lambda, \Sigma, \Xi} \bar{\Psi}_{B}\left[g_{\sigma B} \sigma+g_{\sigma^{\star} B} \sigma^{\star}-\gamma_{\mu} g_{\omega B} \omega^{\mu}\right. \\
& \left.-\frac{1}{2} \gamma_{\mu} g_{\rho B} \tau_{B} \rho^{\mu}-g_{\phi B} \phi^{\mu}\right] \Psi_{B},
\end{aligned}
$$

while the Lagrangian density for non-linear self-interaction of mesons can be expressed as,

$$
\begin{aligned}
\mathcal{L}^{N L}= & -\frac{\kappa_{3} g_{\sigma N} m_{\sigma}^{2}}{6 m_{N}} \sigma^{3}-\frac{\kappa_{4} g_{\sigma N}^{2} m_{\sigma}^{2}}{24 m_{N}^{2}} \sigma^{4} \\
& +\frac{\zeta_{0} g_{\omega N}^{2}}{24}\left(\omega_{\mu} \omega^{\mu}\right)^{2}+\frac{\eta_{1} g_{\sigma N} m_{\omega}^{2}}{2 m_{N}} \sigma \omega_{\mu} \omega^{\mu} \\
& +\frac{\eta_{2} g_{\sigma N}^{2} m_{\omega}^{2}}{4 m_{N}^{2}} \sigma^{2} \omega_{\mu} \omega^{\mu}+\frac{\eta_{\rho} g_{\sigma N} m_{\rho}^{2}}{2 m_{B}} \sigma \rho_{\mu} \rho^{\mu} \\
& +\frac{\eta_{1 \rho} g_{\sigma N}^{2} m_{\rho}^{2}}{4 m_{N}^{2}} \sigma^{2} \rho_{\mu} \rho^{\mu}+\frac{\eta_{2 \rho} g_{\omega N}^{2} m_{\rho}^{2}}{4 m_{N}^{2}} \omega_{\mu} \omega^{\mu} \rho_{\mu} \rho^{\mu}
\end{aligned}
$$

The Lagrangian density for free leptons can be written as,

$\mathcal{L}_{l}^{\text {free }}=\sum_{l=e^{-}, \mu^{-}} \bar{\Psi}_{l}\left[i \gamma^{\mu} \partial_{\mu}-M_{l}\right] \Psi_{l}$.

Here $\Psi_{B}$ is the baryon field; $\tau_{B}$ is the baryon isospin; $\Psi_{l}$ is the lepton field; $M_{B}, M_{l}$ are baryon and lepton masses, respectively; $\sigma, \sigma *, \omega^{\mu}, \phi^{\mu}$, and $\rho^{\mu}$ are meson fields of $\sigma, \sigma *, \omega, \phi$, and $\rho$ mesons, respectively whereas $\omega^{\mu \nu}, \phi^{\mu \nu}$, and $\rho^{\mu \nu}$ are meson field tensors of $\omega, \phi$, and $\rho$, respectively. Note that the corresponding tensors are defined as $\omega^{\mu \nu} \equiv \partial^{\mu} \omega^{\nu}-\partial^{\nu} \omega^{\mu}$; $\phi^{\mu \nu} \equiv \partial^{\mu} \phi^{v}-\partial^{v} \phi^{\mu} ; \rho^{\mu \nu} \equiv \partial^{\mu} \rho^{v}-\partial^{v} \rho^{\mu}$. In EOS numerical calculation, the nucleons coupling constants $\left(g_{\sigma N}, g_{\omega} g_{\rho}\right)$ and all nonlinear parameters $\left(\kappa_{3}, \kappa_{4}, \zeta_{0}, \eta_{1}, \eta_{2}, \eta_{\rho}, \eta_{1 \rho}, \eta_{2 \rho}\right)$ are taken from Refs. [2,24]. The vector part of hyperons coupling constant $g_{\omega H}$ and $g_{\phi H}$ are determined by using SU(6)

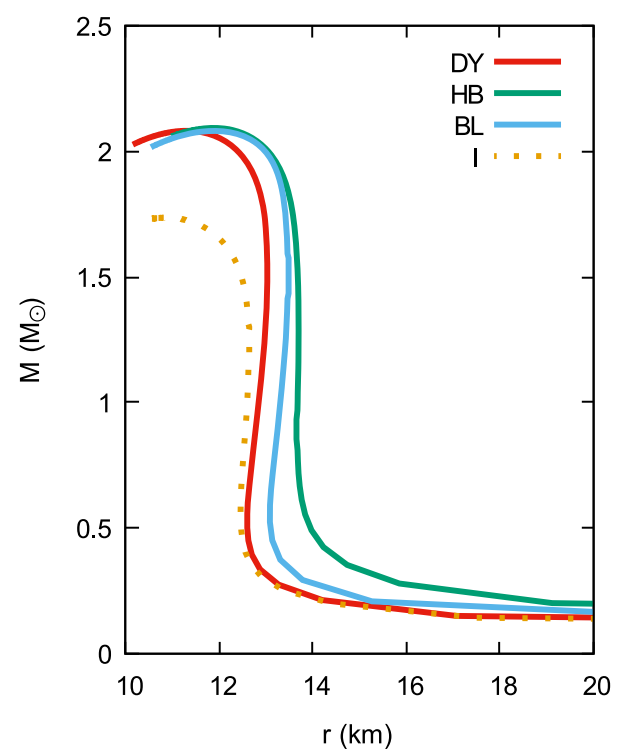

Fig. 1 Mass-radius relation of anisotropic NSs within DY, HB, and BL models. The results of the isotropic model (I) are given to be compared with the anisotropic model parameters i.e. $\lambda, h$, and $c$ 
symmetry [26]. Here $H$ denotes hyperon. The scalar hyperons coupling strengths $g_{\sigma H}$ are calculated from the potential depth of hyperons evaluated at the saturation density of symmetric nuclear matter $\rho_{0}$. Here, we use the values of experimentally potential depth $U_{H}^{(N)}\left(\rho_{0}\right)$ [26] i.e., $U_{\Lambda}^{(N)}=$ $-28 \mathrm{MeV}, U_{\Sigma}^{(N)}=+30 \mathrm{MeV}$, and $U_{\Xi}^{(N)}=-18 \mathrm{MeV}$. The Fermi momentum of each constituent in NSs core is determined using standard $\beta$ stability conditions. The total energy density $(\epsilon)$ of NSs core matter is calculated from the zero component of energy-momentum tensor. The corresponding radial pressure $p$ is calculated from the thermodynamic relation. Note that in the crusts part, we also use crust EOSs data of Ref. [27].

\section{Tolmann-Oppenheimer-Volkoff equations}

To produce static and spherical symmetric anisotropic NSs properties, such as radius $R$ and mass $M$ as well as energy density profile $\epsilon(r)$ and pressure profiles $P(r)$ and $q(r)$, respectively, we need to solve the TOV equation [2]. In the most general case the matter inside a static spherically symmetric stars has a momentum-energy tensor $\left(T^{\mu \nu}\right)$ with anisotropic pressure [2,21,22]. It can be expressed as,

$T^{\mu \nu}=q g^{\mu \nu}+(\epsilon+q) u^{\mu} u^{\nu}+\sigma k^{\mu} k^{\nu}$,

with

$\sigma=p-q$

Here, $\sigma$ is the difference between radial $(p)$ and tangential pressure $(q)$ (known as anisotropic factors), $g^{\mu \nu}$ is space- time metric, $u^{\mu}$ is fluid 4 -velocity, and $k^{\mu}$ is velocity in radial direction where the relation $u^{\mu} k_{\mu}=0$ is fulfilled. And also for the general spherically symmetric static space-time geometry, the metric reads

$d s^{2}=-e^{2 v} d t^{2}+e^{2 \lambda} d r^{2}+r^{2}\left(d \theta^{2}+\sin ^{2} \theta d \phi^{2}\right)$,

to calculate Einstein field tensor $G^{\mu \nu}$. By equating $G^{\mu \nu}$ and $T^{\mu \nu}$, we can obtain the TOV equation for anisotropic NSs as follows, [2]

$\frac{d P}{d r}=-\frac{M \epsilon}{r} \frac{\left(1+\frac{p}{\epsilon}\right)\left(1+\frac{4 \pi r^{3}}{M}\right)}{(r-2 M)}-\frac{2 \sigma}{r}$,
$\frac{d m}{d r}=4 \pi r^{2} \epsilon$.

To generate $P(r), \epsilon(r)$, and $m(r)$ profiles, the TOV equations (Eqs. (10) and (11)) are integrated numerically by using fourth order Runge-Kutta. The calculation start from central where $r \sim 0$ by giving initial value of $P$ such as $P=P_{c}$ then Eq. (10) is integrated up to the edge of the star $(r=R)$ where in the edge, $P(R) \rightarrow 0$. Note that the central mass is set to be $m_{c} \sim 0$ and also Eq. (11) is integrated up to the the edge of the star where the mass of the star is determined from the relation $m(R)=M$.

Note that the source of realistic $\sigma$ comes for many possible microscopic (matter) and macroscopic (geometry) basis in a such non trivial way (please see Ref. [2] and the references therein for details.). Therefore, in this work we only consider some well known models of $\sigma$. In precise, we study and compare 3 well known models of $\sigma$ as

1. DY model [22]: $\sigma \equiv \lambda\left(\frac{2 M}{r}\right) P$
Fig. 2 Radial pressure of anisotropic models NSs and its isotropic (I) for $M=1.4 M_{\odot}$ and $M=M_{\max }$

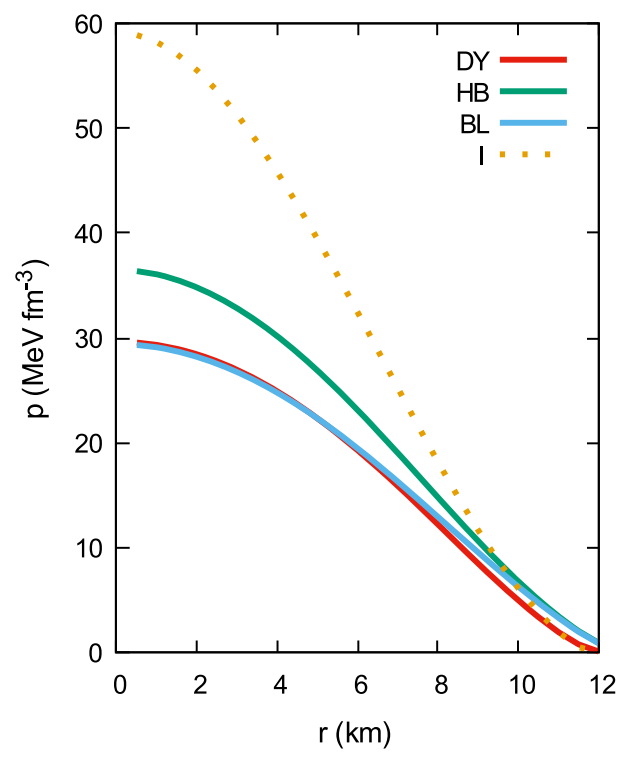

(a) $\mathrm{M}=1.4 \mathrm{M} \odot$

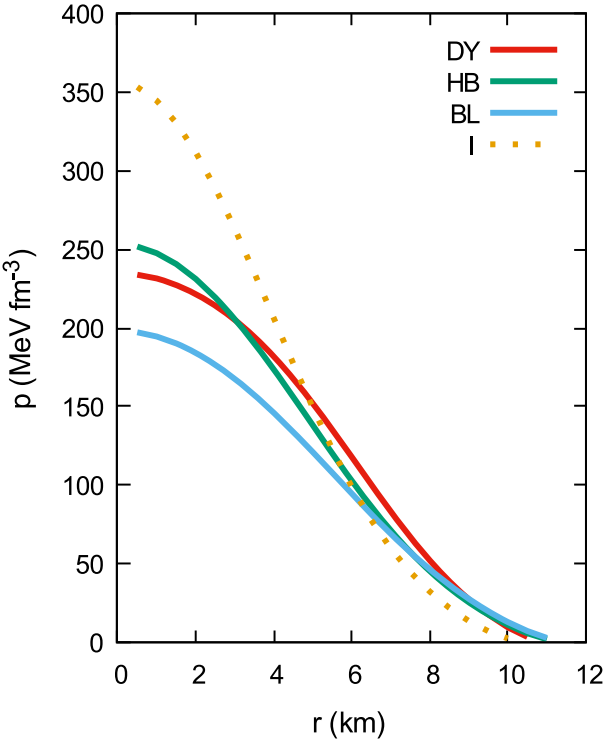

(b) $M=M \max$ 
2. HB model [21]: $\sigma \equiv-\frac{r(1-h)}{2 h} P^{\prime}$

3. BL model [23]: $\sigma \equiv-c(\epsilon-3 P)\left(1-\frac{2 M}{r}\right)^{-1}(\epsilon+P) r^{2}$,

where $\lambda, h$, and $c$ are free parameters of the DY, HB, and BL models, respectively [21-23].

\section{Energy conditions}

The physically acceptable EOS of NSs should obey the perfect fluid's energy conditions [2,4,10-12] in order the NSs is stable. The energy conditions are:
1. $p \geq 0, q \geq 0$, and $\epsilon \geq 0$; meaning that inside the stars, the radial pressure, tangential pressure, and energy density profiles should be positive.

2. $p^{\prime}=d p / d r \leq 0$ and $\epsilon^{\prime}=d \epsilon / d r \leq 0$; meaning that inside the stars, either the gradient of radial pressure or energy density profiles should be negative.

3. $V_{s}^{p} \equiv \partial p / \partial \epsilon \leq 1$ and $V_{s}^{q} \equiv \partial q / \partial \epsilon \leq 1$; meaning that inside the stars, the speed of sound for either radial or tangential pressure profiles are always lower than the speed of light.

4. $\epsilon \geq q$ and $\epsilon \geq p$; meaning that inside the stars, the energy density profile must be higher than radial and tangential pressure profiles.
Fig. 3 Tangential pressure of anisotropic models NSs and its isotropic (I) for $M=1.4 M_{\odot}$ and $M=M_{\max }$

Fig. 4 Energy density of anisotropic models NSs and its isotropic (I) for $M=1.4 M_{\odot}$ and $M=M_{\max }$

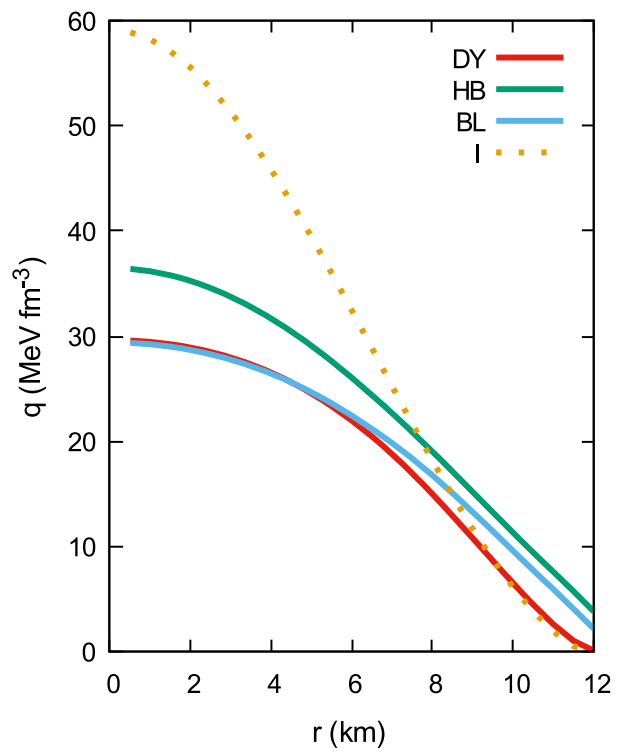

(a) $\mathrm{M}=1.4 \mathrm{M}_{\odot}$

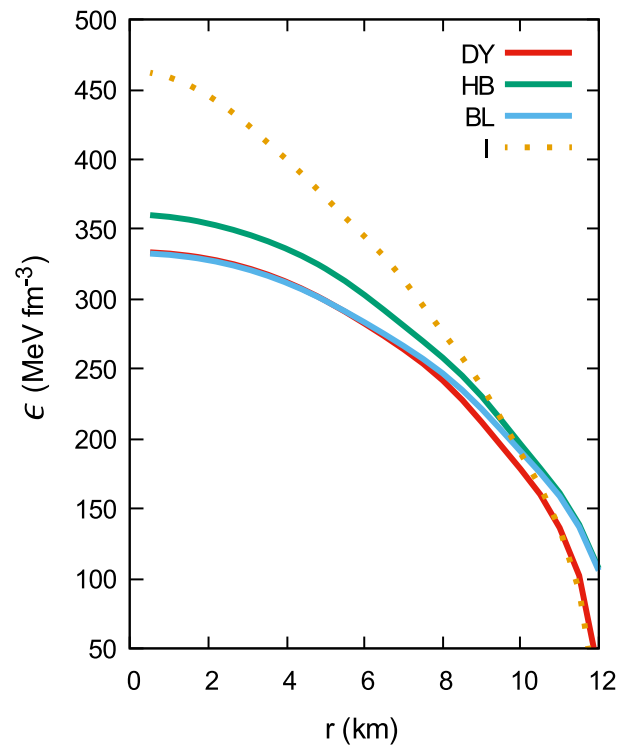

(a) $M=1.4 M_{\odot}$

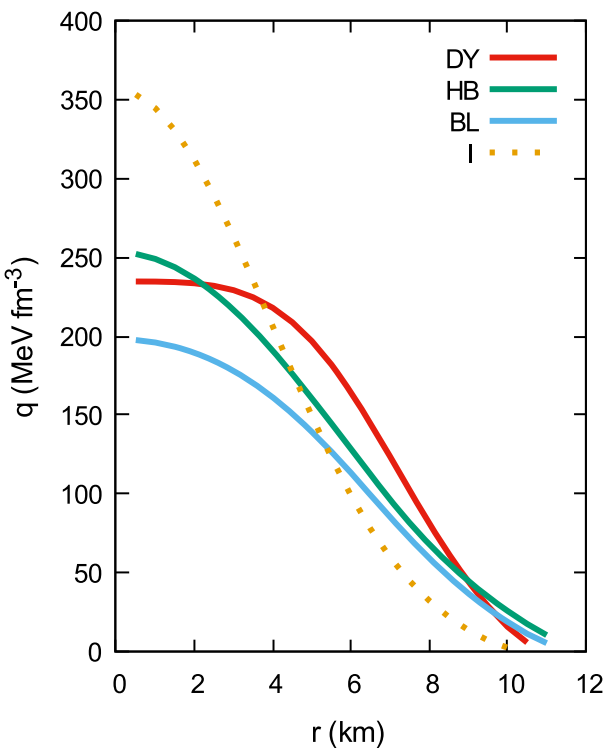

(b) $M=M \max$

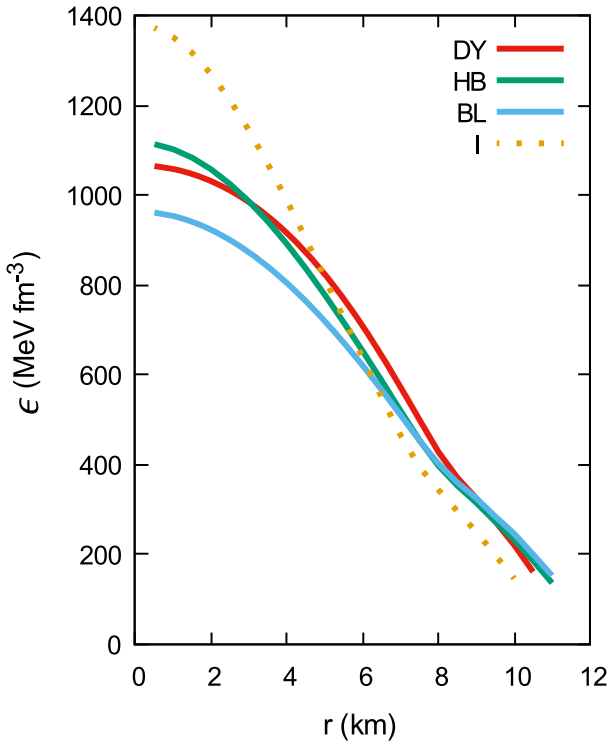

(b) $M=M \max$ 
Table 1 Comparison of energy density, radial and tangential pressure in the center of anisotropic NSs where the unit is in $\mathrm{MeV} \mathrm{fm}^{-3}$

\begin{tabular}{llrrr}
\hline Model & Mass & \multicolumn{1}{c}{$\epsilon_{c}$} & \multicolumn{1}{c}{$p_{c}$} & \multicolumn{1}{c}{$q_{c}$} \\
\hline DY & $1.4 M_{\odot}$ & 333.44 & 29.55 & 29.58 \\
& Max & 1064.82 & 233.83 & 234.63 \\
HB & $1.4 M_{\odot}$ & 360.03 & 36.37 & 36.40 \\
& Max & 1113.57 & 251.77 & 252.12 \\
BL & $1.4 M_{\odot}$ & 332.51 & 29.33 & 29.36 \\
& Max & 961.36 & 197.19 & 197.55 \\
\hline
\end{tabular}

Table 2 Strong energy condition in the center of anisotropic NSs where the unit is $\mathrm{MeV} \mathrm{fm}^{-3}$

\begin{tabular}{llrrr}
\hline Model & Mass $\epsilon_{c}+p_{c}+2 q_{c}$ & \multicolumn{1}{c}{$\epsilon_{c}+p_{c}$} & \multicolumn{1}{c}{$\epsilon_{c}+q_{c}$} \\
\hline DY & $1.4 M_{\odot}$ & 422.15 & 362.99 & 363.02 \\
& Max & 1767.91 & 1298.65 & 1299.45 \\
HB & $1.4 M_{\odot}$ & 469.20 & 396.40 & 396.43 \\
& Max & 1869.59 & 1365.35 & 1365.70 \\
BL & $1.4 M_{\odot}$ & 420.56 & 361.84 & 361.87 \\
& Max & 1553.65 & 1158.55 & 1158.91 \\
\hline
\end{tabular}

5. Strong energy condition: $\epsilon+p+2 q \geq 0, \epsilon+p \geq 0$ and $\epsilon+q \geq 0$.

6. $-1 \leq V_{s}^{q^{2}}-V_{s}^{p^{2}} \leq 1$, where $-1 \leq V_{s}^{q^{2}}-V_{s}^{p^{2}} \leq 0$ and $0 \leq V_{s}^{q^{2}}-V_{s}^{p^{2}} \leq 1$; meaning that the acceptable ranges of the difference between speed of sound in tangential and radial directions are in range between -1 and 1 .

Note that it is reported recently [15], based on anisotropic exact regular solution analysis that if the anisotropy fac- tor disappear it could produce a regular model of perfect fluid which is not physically acceptable. Here we investigate whether the anisotropic EOS of NSs with realistic EOS within the DY, HB, and BL models also obey these energy conditions or not. Our results will complement the finding in Ref. [15].

\section{Results and discussions}

Before we show that the radial and tangential pressure profiles as well as the energy density profile of static and spherically symmetric NSs within DY, HB, and BL anisotropic models and the isotropic model, we show in Fig. 1 the massradius relation of NSs predicted by the $\mathrm{DY}, \mathrm{HB}$, and $\mathrm{BL}$ models using NSs EOS discussed in Sect. 2. The results are compare to that of the isotropic NS model. It can be seen that anisotropic NSs models can reach the maximum mass of NSs $M \geq 2.1 M_{\odot}$ by adjusting the anisotropic free parameter of the corresponding anisotropic model. However, for the same NSs mass, the DY, HB, and BL models predict different corresponding radius. It means anisotropic factor has impact on mass and radius of NSs. From now on, we will focus on the profile of NSs with the $M=1.4 M_{\odot}$ (canonical mass) and $M=M_{\max }$.

Panel (a) and (b) of Fig. 2 shows the radial pressure profiles of anisotropic NSs within DY, HB, and BL models as well as the one of isotropic (I) model for $M=1.4 M_{\odot}$ and $M=M_{\max }$. It can be seen that in both panels that the corresponding radial pressures of the NSs have positive values $(\geq 0)$ everywhere inside the stars. While in Figs. 3 and 4, it is also shown that the tangential pressure and energy density
Fig. 5 The gradient of radial pressure of anisotropic models NSs and its isotropic (I) for $M=1.4 M_{\odot}$ and $M=M_{\max }$

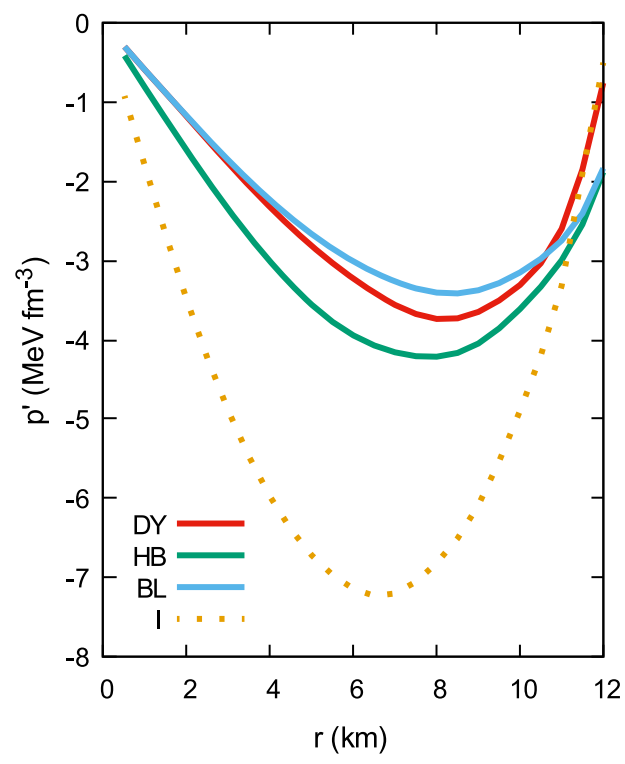

(a) $\mathrm{M}=1.4 \mathrm{M}_{\odot}$

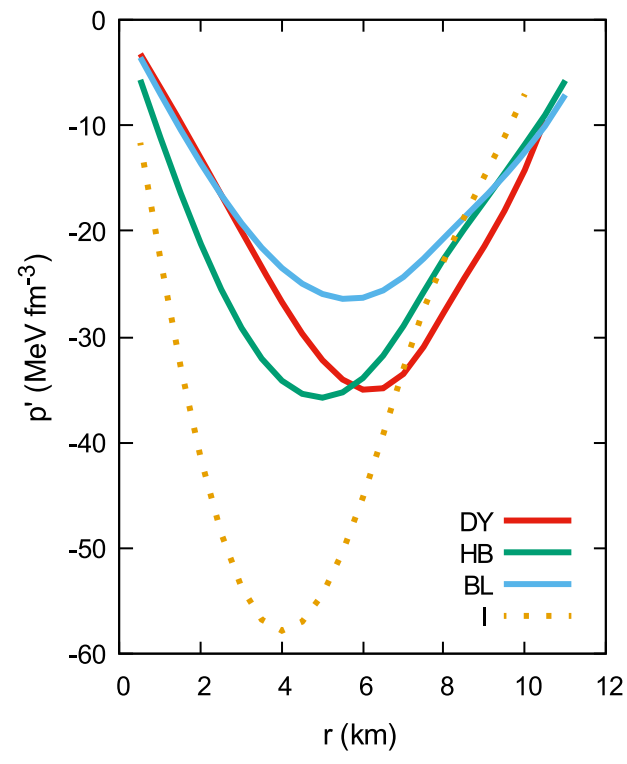

(b) $M=M_{\max }$ 
profiles of all models for $M=1.4 M_{\odot}$ and $M=M_{\max }$, shown a similar behavior to that of radial pressure. The tangential pressure and energy density of the NSs also have positive values $(\geq 0)$ everywhere inside the stars. It can be observed also that the $\epsilon(r), P(r)$, and $q(r)$ profiles, the corresponding energy density, radial and tangential pressures decrease when the radius increases up to $R$. The impact of the anisotropic factor on $q$ appears in the region close to $R$ for the case of anisotropic models and energy density could be non-zero in the region close to $R$. The corresponding values of energy density, radial pressure, and tangential pressure and their combination at the center of anisotropic NS can be seen in Tables 1 and 2. It is obvious from Table 1 that the impact of $\sigma$ is insignificant in center and the corresponding insignificance depends on the NSs mass. It is also obvious that for both NSs masses, $\epsilon_{c} \geq q_{c}$ and $\epsilon_{c} \geq p_{c}$. The later relations actually are also valid for all value in $\epsilon(r), P(r)$, and $q(r)$ profiles. Similarly from Table 2 it can be seen that $\epsilon_{c}+p_{c}+2 q_{c} \geq 0, \epsilon_{c}+p_{c} \geq 0$ and $\epsilon_{c}+q_{c} \geq 0$ and the corresponding values are relative large. These relation also valid for all value in $\epsilon(r), P(r)$, and $q(r)$ profiles. Even we do not show here due to similarity to the ones of anisotropic, we have found that for the isotropic model, the strong energy condition and $\epsilon \geq q$ and $\epsilon \geq p$ relation are also satisfied. It means that these results show that the first, fourth and fifth
Fig. 6 The gradient of energy density of anisotropic models NSs and its isotropic (I) for $M=1.4 M_{\odot}$ and $M=M_{\max }$

Fig. 7 The sound of speed for radial pressure of anisotropic models NSs and its isotropic (I) for $M=1.4 M_{\odot}$ and $M=M_{\max }$

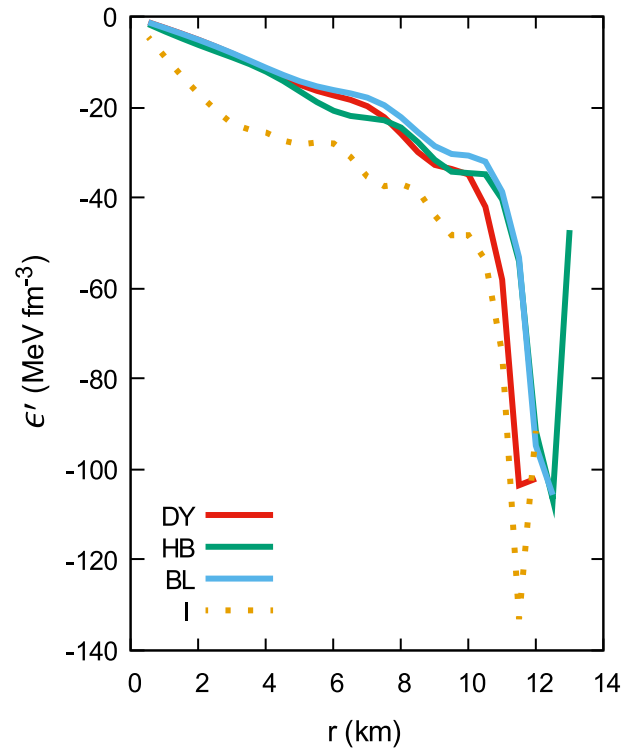

(a) $M=1.4 M_{\odot}$

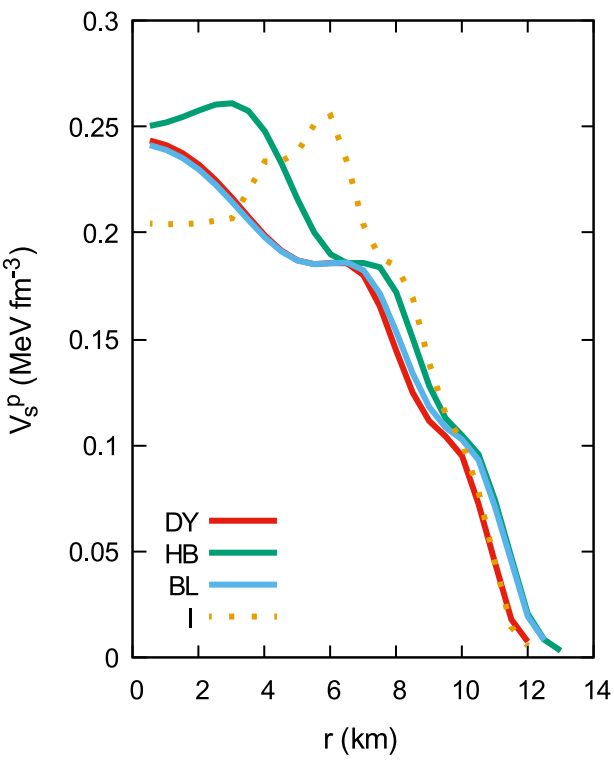

(a) $\mathrm{M}=1.4 \mathrm{M}_{\odot}$

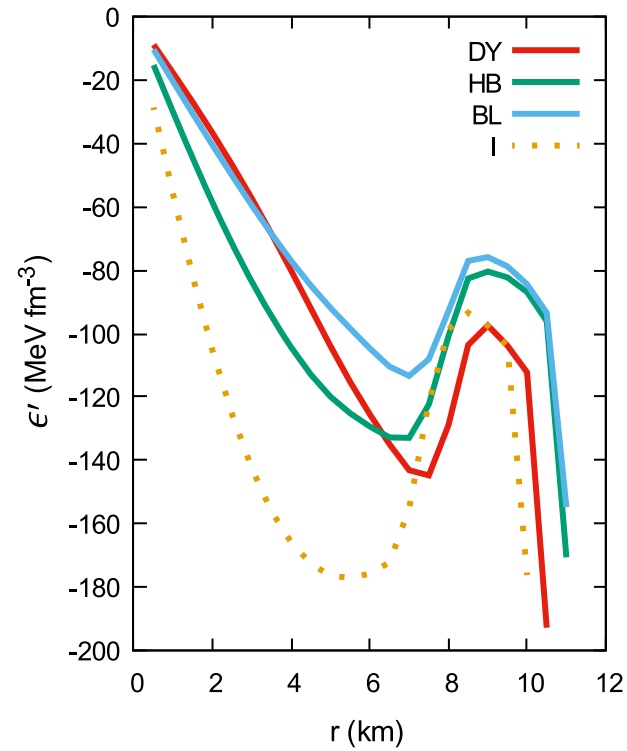

(b) $M=M \max$

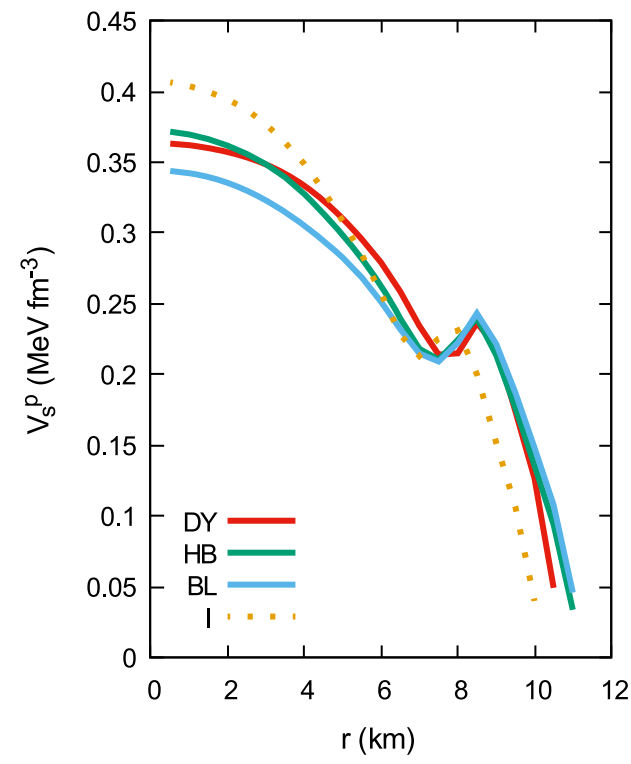

(b) $M=M_{\max }$ 
conditions of perfect fluid's energy conditions are fulfilled for all models.

Figure $5 \mathrm{a}, \mathrm{b}$ show that the gradient of radial pressure of all NSs models for $M=1.4 M_{\odot}$ and $M=M_{\max }$. It is obvious that the gradient of radial pressure profiles predicted by all models have negative values $(\leq 0)$ with the maximum effect appears on the middle of the distribution and for larger NSs mass. Figure $6 \mathrm{a}, \mathrm{b}$ show that the gradient of energy density of all models for $M=1.4 M_{\odot}$ and $M=M_{\max }$. It can be seen that the gradient of radial energy density profiles predicted by all models have negative values $(\leq 0)$ with the maximum effect appears for larger NSs mass. The different
EOS structure between core and crust is reflected in the gradient of radial energy density profiles. These results show that the second condition of perfect fluid's energy conditions are fulfilled, where $p^{\prime}=d p / d r \leq 0$ and $\epsilon^{\prime}=d \epsilon / d r \leq 0$ inside the NSs by all models of NSs.

Figure 7a, b as well as Fig. 8a, b show the speed of sound for radial and tangential pressure profiles for all NSs models for $M=1.4 M_{\odot}$ and $M=M_{\max }$. Even the profile trend of radial and tangential speed of sound due to anisotropic effect is different in anisotropic models, it is obvious that the range of both speed of sound profiles is still $\leq 1$ is satisfy by all models. These results show that the third condition of
Fig. 8 The speed of sound tangential pressure of anisotropic models NSs and its isotropic (I) for $M=1.4 M_{\odot}$ and $M=M_{\max }$
Fig. 9 The difference in speed of sound of anisotropic models NSs and its isotropic (I) for $M=1.4 M_{\odot}$ and $M=M_{\max }$

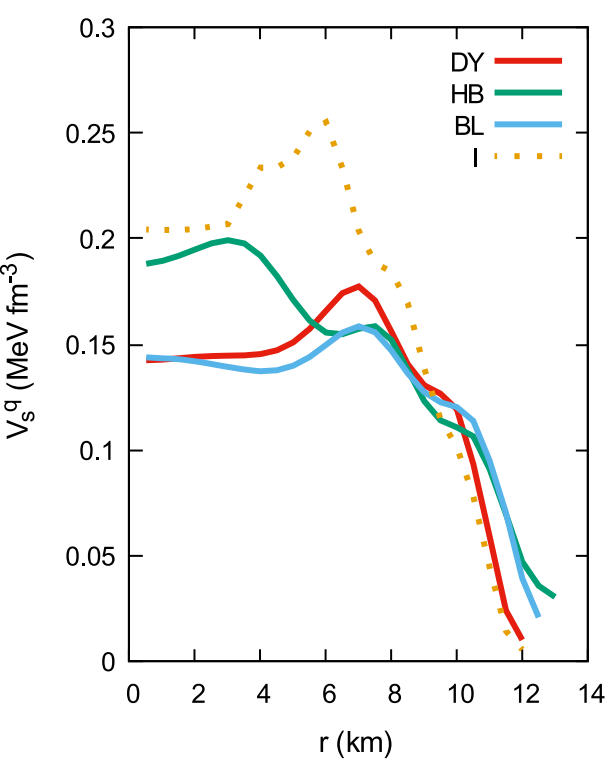

(a) $\mathrm{M}=1.4 \mathrm{M}_{\odot}$

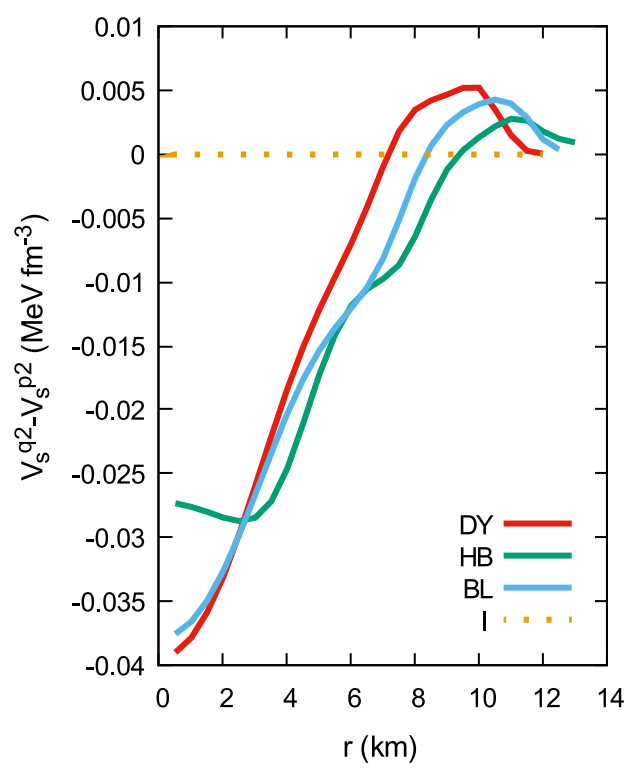

(a) $\mathrm{M}=1.4 \mathrm{M}_{\odot}$

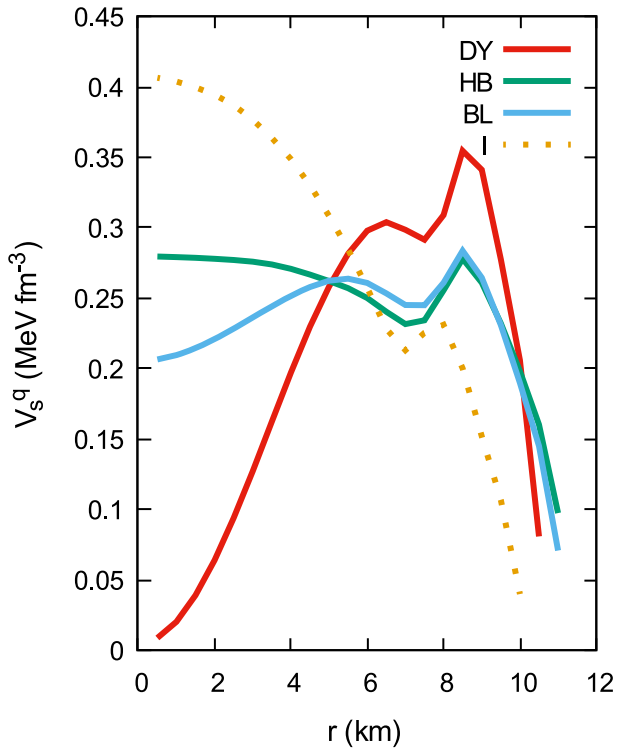

(b) $\mathrm{M}=\mathrm{M}_{\max }$

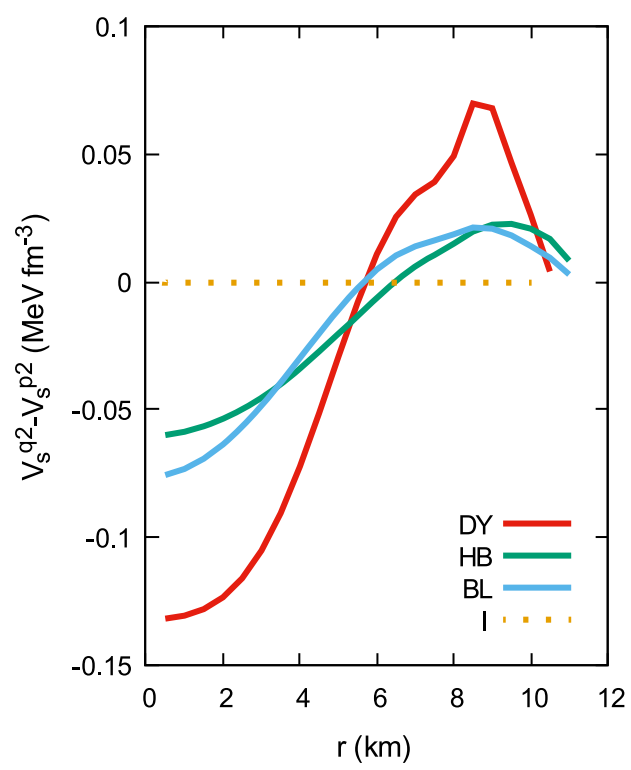

(b) $M=M \max$ 
perfect fluid energy conditions, where $V_{s}^{p} \equiv \partial p / \partial \epsilon \leq 1$ and $V_{s}^{q} \equiv \partial q / \partial \epsilon \leq 1$; is also fulfilled by all models. Figure 9a, $b$ show that the difference in sound of speed for tangential and radial pressure profiles of all models for $M=1.4 M_{\odot}$ and $M=M_{\max }$. It is obvious that the sixth condition from perfect fluid energy conditions is also fulfilled by all models i.e., the difference between sound of speed in tangential and radial directions inside the stars are in the range between -1 and 1 . We need to note that the the difference between sound of speed in tangential and radial directions change the sign around the middle of the stars due to difference in composition and the structure of realistic EOS of NSs used for the case of anisotropic models.

\section{Conclusion}

In this work, we calculate the radial and tangential pressures and the energy density profiles of NSs with $M=1.4 M_{\odot}$ and $M=M_{\max }$ using realistic EOS predicted by relativistic mean-field (RMF) model including hyperons for isotropic and three different anisotropic pressure models. The used anisotropic models are the one of Doneva and Yazadjiev [21], the one of Herrera and Barreto [22], and the one of Bowers and Liang [23]. We have shown that the all of energy conditions of perfect fluid are satisfied not only by anisotropic NSs profiles but also the one of isotropic NSs. The anisotropic NS results are compatible with the ones from the analytic solution obtained by the authors of Ref. [15]. However, for the isotropic NSs, we have found that the energy density and the speed of sound at origin are also positive at the same time.

Acknowledgements A. Sulaksono is funded by Universitas Indonesia, Q1Q2 Grant no. NKB-0267/UN2.R3.1/HKP.05.00/2019.

Data Availability Statement This manuscript has no associated data or the data will not be deposited. [Authors' comment: The data will be deposited privately by the author for the next research.]

Open Access This article is distributed under the terms of the Creative Commons Attribution 4.0 International License (http://creativecomm ons.org/licenses/by/4.0/), which permits unrestricted use, distribution, and reproduction in any medium, provided you give appropriate credit to the original author(s) and the source, provide a link to the Creative Commons license, and indicate if changes were made.

Funded by SCOAP ${ }^{3}$.

\section{References}

1. L. Herrera, N.O. Santos, Phys. Rep. 286, 53 (1997)

2. A. Sulaksono, Int. J. Mod. Phys. E 24, 1550007 (2015)

3. K. Dev, M. Gleiser, Gen. Relativ. Gravit. 34, 1793 (2002)

4. H. Abreu, H. Hernández, L.A. Núñez, Class. Quantum Gravity 24, 4631 (2007)

5. A. Wojnar, H. Velten, Eur. Phys. J. C 76, 697 (2016)

6. J. Ovalle, Phys. Rev. D 95, 104019 (2017)

7. H. Hernández, L.A. Núñez, A. Vásquez-Ramírez, Eur. Phys. J. C 78, 883 (2018)

8. V. Folomeev, Phys. Rev. D 97, 124009 (2018)

9. S.K. Maurya, A. Banerjeey, S. Hansrajz, Phys. Rev. D 97, 044022 (2018)

10. M.K. Mak, T. Harko, Proc. R. Soc. Lond. Ser. A 459, 393 (2003)

11. C. Kolassis, N.O. Santos, D. Tsoubelis, Class. Quantum Gravity 5, 1329 (1988)

12. A. Di Prisco, L. Herrera, V. Varela, Gen. Relativ. Gravit. 29, 1239 (1997)

13. G. Raposo, P. Pani, M. Bezares, C. Palenzuela, V. Cardoso, Phys. Rev. D 99, 104072 (2019)

14. A. Urbano, H. Vermäe, JCAP 1904, 011 (2019)

15. G. Estevez-Delgado, J. Estevez-Delgado, Eur. Phys. J. C 78, 673 (2018)

16. F. Özel, P. Freire, Ann. Rev. Astron. Astrophys. 54, 401 (2016)

17. B.A. Li, P.G. Krastev, D.H. Hua, N.B. Zhang, Eur. Phys. J. A 55, 117 (2019)

18. L. Rezzola, K. Takami, Phys. Rev. D 93, 124051 (2016)

19. E. Annala, T. Gorda, A. Kurkela, A. Vuorinen, Phys. Rev. Lett. 120, 172703 (2018)

20. E.R. Most, L.R. Weih, L. Rezzolla, J. Schaffner-Bielich, Phys. Rev. Lett. 120, 261103 (2018)

21. D.D. Doneva, S.S. Yazadjiev, Phys. Rev. D 85, 124023 (2012)

22. L. Herrera, W. Barreto, Phys. Rev. D 88, 084022 (2013)

23. R.L. Bowers, E.P.T. Liang, Astrophys. J. 188, 657 (1974)

24. A. Sulaksono, B.K. Agrawal, Nucl. Phys. A 895, 44 (2012)

25. B.K. Agrawal, A. Sulaksono, P.-G. Reinhard, Nucl. Phys. A 882, $1(2012)$

26. J. Schaffner-Bielich, A. Gal, Phys. Rev. C 62, 034311 (2000)

27. T. Miyatsu, S. Yammamuro, K. Nakasaki, Astrophys. J 777, 4 (2013) 